Convergences francophones 3.1 (2016) : 10-21

http://mrujs.mtroyal.ca/index.php/cf/index

\title{
Les anecdotes du suttisme- image d'une altérité féminine ambiguë
}

\author{
Devika Vijayan \\ University of Calgary
}

L'Inde est au XVII ${ }^{\mathrm{e}}$ siècle un ailleurs géographique et culturel qui interroge le public européen, qu'informent récits et relations de voyages de plus en plus nombreux et accessibles. Leurs auteurs, cherchant à allier l'utile et l'agréable, dressent l'inventaire du continent indien tout en dynamisant leurs textes de plusieurs manières parmi lesquelles l'anecdote. La définition littéraire de l'anecdote comme étant un "récit succinct d'un petit fait curieux » (Trésor de la langue française) fait ressortir quelques éléments essentiels. D'abord, en ce qui concerne la composante narrative, l'anecdote est un récit, mais c'est un récit qui est bref dans sa forme. En ce qui concerne le sujet, il doit piquer l'attention et éveiller la curiosité du lecteur. De ce fait, il n'est pas étonnant que les récits de voyage soient un terrain d'élection privilégié de l'anecdote car c'est souvent au retour d'un séjour que nous racontons des petites histoires qui ont retenu notre attention. Le voyageur relate souvent les faits surprenants dont il a été témoin ou dont il a entendu parler. En ce qui concerne les récits viatiques aux Indes orientales, il y a de nombreux exemples de ces faits surprenants qui sont issus de la rencontre des cultures orientales et occidentales et qui font de l'anecdote un des lieux où s'exprime l'altérité. Une de ces pratiques qui ne cessait d'étonner le public européen est celui du «suttisme $»^{1}:$ l'immolation des veuves hindoues sur le bûcher funéraire de leurs maris. Certains chercheurs, comme Pompa Banerjee, pensent que les voyageurs utilisent les récits anecdotiques sur ce rite comme tremplin pour promouvoir leurs idées sur une altérité religieuse barbare. ${ }^{2}$ Comme preuve, elle se réfère à de nombreux exemples dans les récits viatiques vers les Indes orientales qui font ressortir toute la tragédie et l'horreur associées à cette coutume.

Dans cet article nous remettons en question cette hypothèse en examinant le cas spécifique des voyageurs français aux Indes orientales au XVII et XVIII ${ }^{\mathrm{e}}$ siècles. En analysant les récits anecdotiques, nous démontrerons que ce rite met plutôt en scène un discours sur une altérité féminine fracturée, ce qui est largement symptomatique de l'image ambiguë de la femme durant l'Ancien Régime. Il convient donc de ne pas accuser trop rapidement les voyageurs d'avoir lancé une campagne qui présente le sous-continent indien comme étant « le grand contraire complémentaire » (Saïd 9) du monde occidental.

\section{« Sati » : un mot à l'origine ambigüe}

\footnotetext{
${ }^{1}$ Nous empruntons le terme "suttisme» de Catherine Weinberger Thomas dans Cendres d'immortalité. La crémation des veuves en Inde. Paris : Seuil, 1996.

${ }^{2}$ Dans son livre Burning women: widows, witches and early modern European travellers in India, New York, Palgrave Macmillan, 2003, Banerjee analyse plusieurs discours qui découlent de la représentation de la sati dans les relations de voyage vers les Indes orientales. Ici nous remettons en cause l'argument principal de son livre.
} 
On ignore l'origine exacte de ce terme. Il demeure perdu dans la nuit des temps. John Holwell (45-106), qui a vécu en Inde au XVIII ${ }^{\mathrm{e}}$ siècle, prétend que le rite est prescrit aux femmes hindoues par la loi divine. Afin d'expliquer le raisonnement derrière cette pratique, il se réfère aux Védas, livre sacré des hindous, où l'on raconte l'histoire des épouses du dieu Brahma. Elles étaient si malheureuses après son décès qu'elles décidaient de se sacrifier sur le bûcher funéraire de leur mari et de l'accompagner au Paradis. Holwell croit que cet exemple divin a été suivi par le peuple. Les indologues, cependant, ne trouvent aucune mention de cette histoire dans les Védas et Alain Daniélou confirme ce propos dans son livre La civilisation des différences. Selon lui « la pratique de la sati est inconnue des textes anciens » (Daniélou 45) $)^{3}$. Catherine Weinberger-Thomas, dans son livre Cendres d'immortalité, démontre que le mot « sati » dérivé du sanscrit «as » (être), donne sât (étant) au participe présent et sâti au féminin. Par ajout sémantique, " sât » est associé à l'idée de vertu. Le mot « sati » désigne donc l'épouse qui est vertueuse, chaste et fidèle et non le rite de la crémation des veuves. Quel que soit l'origine de ce mot, la sati exerce sur la psyché occidentale une fascination sans pareille ${ }^{4}$ et ce rite trouve mention dans presque toutes les relations de voyage des XVII et XVIII ${ }^{\mathrm{e}}$ siècles.

\section{Le suttisme : discours sur l’altérité barbare}

Il est indéniable que certains de nos voyageurs s'insurgent contre cette horrible coutume des veuves qui s'immolent sur le bûcher de leurs maris. François Bernier, par exemple, condamne ce rite qu'il qualifie d' " horribles spectacles » ou de « coutumes barbares » (309). Selon lui, ce sont les brahmanes ou les prêtres hindous qui orchestrent cet holocauste. De plus, ils obligent les veuves à s'y astreindre, et ce, parfois contre leur gré:

Il me souvient entre autres que je vis brûler à Lahore une femme qui était très belle et qui était encore toute jeune : je ne crois pas qu'elle eût plus de douze ans. Cette pauvre petite malheureuse paraissait plus morte que vive à l'approche du bûcher ; elle tremblait et pleurait à grosses larmes, et cependant trois ou quatre de ces bourreaux, avec une vieille qui la tenait par-dessous l'aisselle, la poussèrent et la firent asseoir sur le bûcher et, de crainte qu'ils avaient qu'elle ne s'enfuit ... ils lui lièrent les pieds et les mains, mirent le feu de tous côtés et la brûlèrent toute vive (314)

Bernier aurait assisté au déroulement du sacrifice sans réagir. La jeune veuve d'à peine douze ans tremblait de peur, mais elle est poussée dans le brasier par les brahmanes et une vieille femme. L'utilisation des adverbes «très » ou « tout » et l'accumulation des adjectifs «pauvre petite malheureuse» sert à souligner la

${ }^{3}$ Voir aussi le livre d'Arvind Sharma Sati : Historical and Phenomenological Essays. Delhi : Motilal Banarsidas, 1988.

${ }^{4}$ Dans son livre Pompa Banerjee dénombre au moins quarante-trois témoignages de suttisme pour la période entre 1500-1723. 
jeunesse de la fillette et sa détresse. La victime est d'ailleurs enchaînée au bûcher de son mari avant que l'on n'attise de nouveau le feu. Les brahmanes, quant à eux, sont qualifiés de "bourreaux », transformant cette anecdote descriptive en un véritable spectacle d'horreur. Cette histoire a sans doute fait peur à un nombre considérable de lecteurs et la description minutieuse a vraisemblablement marqué l'imaginaire occidental.

L'impact de François Bernier sur ses contemporains français et européens est à juger par les rééditions successives de son livre en France et ailleurs. Il fait imprimer son Histoire de la dernière révolution des états du grand Mogol en 1670 et devient aussitôt le plus célèbre de tous les voyageurs de son temps. La traduction anglaise des mémoires de Bernier paraît presque aussitôt en 1671. Johann Wilhelm Serlin fournit, en 1672, une version allemande, et en 1675 l'Istoria della ultima revoluzione delli stati del gran mogor dell sr. Bernier est publiée en Italie. François Bernier, de son vivant, devient ainsi un personnage européen. Le grand nombre d'éditions posthumes prouve que son influence sur les générations suivantes a été considérable. L'ampleur de la diffusion de l'œuvre de Bernier n'est certainement pas le seul critère pour juger de son impact sur le public de son temps. Son récit, comme nous l'avons dit, avait enflammé l'imagination occidentale. Il est celui grâce à qui le personnage du «grand mogol» s'installe dans la littérature européenne. Raymond Schwab le précise bien lorsqu'il écrit «Bernier est le premier exemple de ces actifs intermédiaires entre indianisme et littérature ». En 1675, le dramaturge anglais John Dryden écrit même une tragédie intitulée Aurangzeb $b^{5}$ qui s'inspire du livre de François Bernier. Ainsi dans l'acte V de cette tragédie, on voit Mélésinda, l'épouse du prince Morat (fils cadet de l'empereur) se sacrifier sur le bûcher de son mari.

Dans l'anecdote que Jean Thévenot nous fournit sur ce rite, il s'insurge contre les brahmanes. Il explique aux lecteurs les véritables intentions de ces derniers qui obligent les femmes hindoues à s'immoler par le feu. Selon lui, la meilleure partie des objets et des bijoux dont sont parées les victimes revient de droit aux prêtres si elles meurent. C'est pour cette raison que Thévenot qualifie les brahmanes de « tyrans ». La focalisation de la scène, si on la compare à la description de Bernier, se déplace cette fois-ci des sentiments de l'épouse à l'horreur du spectacle vu de l'extérieur et décrit sur fond sonore.

Tout comme ses prédécesseurs, Anquetil-Duperron, qui voyage dans le sous-continent au XVIII ${ }^{\mathrm{e}}$ siècle, condamne le « suttisme » :

Je m'arrêtai à quelque distance de cet endroit pour voir un spectacle qui a été décrit par plusieurs voyageurs. C'était une jeune femme marâthe que la tyrannie de la coutume obligeait de se brûler avec le cadavre de son mari. Les brandons allumés, le bruit des tambours, le son clapissant des flûtes et les cris des assistants ajoutaient à l'horreur de la cérémonie (268)

\footnotetext{
${ }^{5}$ Le dramaturge, semble-t-il, a écrit la pièce après avoir lu la traduction anglaise de Bernier en 1671. L'historien anglais Vincent Smith est de l'opinion que plusieurs passages de cet ouvrage ne sont que des paraphrases du texte de Bernier.
} 
Le voyageur présente la femme comme étant une personne qui n'a aucunement le droit d'exprimer ses opinions. L'utilisation des mots comme «obliger» et «tyrannie » démontrent qu'elle est prisonnière des coutumes du pays. Les musiciens se chargent de masquer les cris de celle qui est vouée à la mort alors que la foule en délire assiste au monstrueux sacrifice.

Il est évident que le rite de l'immolation des veuves est un thème récurrent dans les relations de voyage vers les Indes orientales. Les recherches consacrées à la littérature viatique, en particulier celles menées par Marie-Christine GomezGéraud et Frank Lestringant, révèlent, la nature profondément topique des récits de voyage. En effet, les voyageurs étaient souvent « contraints d'ajouter des passages fictifs pour combler les manques d'une expérience singulière » (Lestringant, XLVLX). Alors comment justifier les choix éditoriaux qui continuent à propager les anecdotes itératives du sous-continent? La réponse, selon certains critiques comme Edward Saïd, réside dans la volonté de démontrer que l'univers occidental est le contraire de l'univers oriental. Dans son livre Orientalism : Western conceptions of the Orient, Saïd parle de deux sentiments opposés qui se sont développés dans le discours orientaliste. D'une part, il y a un sentiment d'attirance pour l'Orient et, d'autre part, un sentiment de rejet qui se traduit par l'attribution aux Orientaux de certains vices comme la paresse, l'ignorance et la sensualité. Transmis de génération en génération, ces discours entretiennent le mythe orientaliste. L'Orient a d'ailleurs permis à l'Europe de se définir par contraste en lui permettant de « préciser son identité en se démarquant de l'Orient» (9).

Alors qu'est-ce qui fait que l'autre est mauvais ? Selon Said, c'est parce qu'il est autre. La différence dérange et perturbe le monde du voyageur. Le « suttisme », qui est le spectacle de l'horreur par excellence, devient ainsi la preuve flagrante de ce mal et raconter ce rite n'est donc que la confirmation de ce fait par le voyageur. Il va sans dire que ces anecdotes sont aujourd'hui l'objet d'attaques virulentes de certains orientalistes. La question qu'ils se posent est la suivante : pour traduire une altérité religieuse le voyageur a souvent recours à la comparaison qui, comme le dirait François Hartog, consiste à «ramener l'autre au même ». Alors pourquoi les voyageurs n'ont-ils pas décrit cette pratique indienne en s'inspirant des exécutions des sorcières qui ont connu leur apogée aux $\mathrm{XVI}^{\mathrm{e}}$ et XVII ${ }^{\mathrm{e}}$ siècles ? Comme se demande Pompa Banerjee: « When European witnesses of sati watched a woman burn... why didn't they seize the analogy of burning witches in their own countries in order to better explain the unfamiliar event of sati to their audiences at home? » (Burning Women 35).

Pourquoi ce lien est-il occulté par les observateurs européens? Nous sommes de l'avis que les voyageurs français ne parvenaient pas à associer cette pratique à une qualité ou à une "valeur » occidentale. Ils pouvaient cependant établir des parallèles lorsqu'il était question des femmes qui acceptaient ce sacrifice avec bravoure. Les femmes hindoues seraient alors différentes des sorcières, mais pourraient être associées à un personnage comme Jeanne d'Arc. En effet, François Bernier établit des parallèles entre le « suttisme » et la cruauté du sacrifice humain dans la religion grecque. Il cite un passage célèbre de Lucrèce rappelant le sacrifice d'Iphigénie, fille d'Agamemnon et de Clytemnestre, qui est décapitée à l'autel de la déesse Trivia pour aider la flotte grecque à atteindre les côtes de Troie. 
La religion souvent enfanta crimes et sacrifices. Ainsi, en Aulide, l'autel de la vierge Trivia, du sang d'Iphigénie fut horriblement souillé par l'élite des Grecs ....

Combien la religion suscita des malheurs! (Bernier 499).

Ce passage est aussi cité par le comte de Modave. Le sacrifice humain devient ainsi un emblème « des choses bien barbares et bien cruelles » (Bernier 315) dans toutes les religions et non pas uniquement dans l'hindouisme.

Qui plus est, la comparaison du «suttisme» avec la persécution des sorcières est rarissime. Elle n'était pourtant pas inexistante comme l'affirme Banerjee. Parmi les voyageurs de notre corpus, François Bernier nous présente ce rite sous un angle qui rapproche la coutume indienne du châtiment des femmes occidentales accusées d'avoir pactisé avec le diable. Dans l'anecdote suivante, Bernier essaie d'empêcher la femme de son ami de son intention de se sacrifier :

Un de mes amis nommé Bendidas ... vint à mourir d'une fièvre étique dont je l'avais traité plus de deux ans. Sa femme résolut aussitôt de se brûler avec le corps de son mari ;... Les parents, n'ayant rien pu gagner sur son esprit par tout ce qu'ils lui avaient pu représenter, s'avisèrent de me prier de l'aller trouver...comme ancien ami de la maison. Je vis en entrant un sabbat de sept ou huit vieilles horribles à voir, avec quatre ou cinq vieux infatués et écervelés de brahmanes qui criaient tous par reprises et en battant des mains à l'entour du mort, et la femme tout échevelée, le visage pâle, les yeux secs et étincelants, qui était assise et qui criait en battant aussi des mains en cadence comme les autres aux pieds de son mari (369)

Il est intéressant de noter que pour traduire cette pratique indienne pour le public français, Bernier ait choisi le vocabulaire de la sorcellerie. Remarquons les termes comme « le sabbat des vieilles » et tout le tintamarre qui accompagne ce rituel. La description de la veuve " tout échevelée » et ayant « le visage pâle, les yeux secs et étincelants ", son aspect diabolique, tout montre une convergence de discours où la veuve indienne prend les traits des sorcières condamnées en Europe. Pour ces raisons, on aurait tort de conclure que les voyageurs utilisent les récits anecdotiques sur le « suttisme » pour promouvoir l'idée d'une altérité religieuse barbare, voire une image stéréotypée des brahmanes. À notre avis, certains de nos voyageurs parviennent bien à dédramatiser cette pratique.

\section{Le suttisme : discours sur la femme perverse}

Si ce rite est vu comme étant le synonyme d'une altérité hindoue barbare, il cohabite avec un autre discours, juridique cette fois-ci, qui essaie de le justifier en le présentant comme une protection contre les intrigues des femmes empoisonneuses. De nouveau, c'est François Bernier qui nous raconte l'anecdote d'une femme mariée qui tombe éperdument amoureuse de son voisin et décide ainsi d'empoisonner son mari : 
Convergences francophones 3.1 (2016) : 10-21

http://mrujs.mtroyal.ca/index.php/cf/index

Je n'étais pas présent à l'action ... C'est d'une femme qui avait quelques amourettes avec un jeune mahométan, son voisin, qui était tailleur et joueur de tambourin. Cette femme, dans l'espérance qu'elle avait que le jeune homme l'épouserait, empoisonna son mari et s'en vint tout aussitôt dire à son tailleur qu'il était temps de partir et de s'enfuir ensemble comme ils avaient projeté, ou qu'autrement elle serait honnêtement obligée de se brûler ; le jeune homme, qui eut crainte de s'embarrasser dans quelque mauvaise affaire, la refusa tout court, mais la femme, sans s'émouvoir ni s'étonner autrement, fut trouver ses parents, les avertit de la mort subite de son mari et leur protesta hautement qu'elle ne voulait point survivre et qu'elle se voulait brûler avec lui ... Les parents, bien contents d'une si généreuse résolution font aussitôt une fosse, la remplissent de bois, mettent le corps sur le bûcher et allument le feu. Tout étant ainsi préparé, la femme va embrassant et disant adieu à tous ses parents [...] Cette furie de femme, étant venue proche de ce jeune homme, fit semblant de lui vouloir aussi dire adieu comme aux autres, mais au lieu de l'embrasser doucement, elle le prend de toute sa force au collet...le fait tomber avec elle la tête la première dedans où ils firent bientôt dépêchés (Bernier 311)

Comment expliquer cette anecdote de la femme empoisonneuse ? Pour pouvoir bien répondre à la question, il faut faire une petite digression et s'attarder sur la question épineuse du statut de la femme durant l'Ancien Régime. Pour l'histoire des femmes, cette période est paradoxale et ambigüe et, pour bien la comprendre, les historiens ont pendant longtemps étudié les personnalités féminines de la Renaissance triomphante. Ainsi, on ne pouvait que partager l'enthousiasme de Rabelais pour une époque où les femmes et les filles aspiraient à la bonne doctrine (205), de l'admiration d'un Bussy pour « ces femmes polies et civiles qui font tout le charme de la cour » (Berriot-Salvadore 12). De ce point de vue, le XVI ${ }^{\mathrm{e}}$ siècle marque bien un moment décisif dans l'évolution de la condition féminine. L'influence italienne avait profondément transformé les mœurs aristocratiques. Il faut cependant souligner que les textes littéraires sont forts sélectifs et mettent toujours en scène une élite sociale. Ils ignorent les paysannes, les ouvrières et artisanes, c'est-à-dire une grande partie de la population féminine française. À ces oublis, viennent s'ajouter les perceptions et représentations de la femme véhiculée par la tradition littéraire. Les allusions à l'imperfection féminine, déjà développées dans les écrits scientifiques de Claude Gallien seront en partie reprises durant la Renaissance. Cette théorie repose sur la définition de tempéraments humains en fonction de la prédominance des humeurs corporelles. Ainsi le caractère froid et humide de la femme explique sa faiblesse et son instabilité et elle cède aussi plus aisément aux plaisirs immodérés de la chair. Le livre de Gallien sera l'objet de nombreuses traductions et de rééditions et en 1659, le médecin français, Marin Cureau de la Chambre s'en inspire largement pour écrire son Art de connoistre les hommes. Dans ce livre, il affirme que « la femme est froide et humide » et il ajoute :

... parce qu'elle est froide, il faut qu'elle soit faible et ensuite timide, pusillanime, soupçonneuse, rusée, dissimulée, flatteuse, menteuse, aisée à offenser, vindicative...Et parce qu'elle est humide, il faut qu'elle soit 
Convergences francophones 3.1 (2016) : 10-21

http://mrujs.mtroyal.ca/index.php/cf/index

mobile, légère, infidèle, impatiente, facile à persuader, pitoyable, babillarde (50)

Cette vision négative du sexe féminin durera par ailleurs jusqu'au XVIII ${ }^{\mathrm{e}}$ siècle. ${ }^{6}$ Un discours stéréotypé à propos de la femme se forme et on lui accole des épithètes comme « inconstante » ou même " fragile ». Pour les chercheurs comme Edward Saïd et Pompa Banerjee, la femme indienne ou orientale se trouve à la charnière de deux discours : celui sur la femme et celui sur l'Orient, ce qui fait qu'elle est perverse. Ainsi, Pyrard de Laval est sensible à l'ardeur sexuelle de la femme du sous-continent indien car il pense que «trois hommes ne suffiraient pas à une femme, tant elles sont impudiques ». Rappelons également les récits anecdotiques sur les amourettes de la princesse Raushanara Begum dans la relation de JeanBaptiste Tavernier et celle de François Bernier. La femme avide de plaisirs, est alors freinée par la loi du sérail qui est contraire à son tempérament. Au XVIII siècle, Anquetil-Duperron raconte une anecdote à propos des bayadères ou danseuses hindoues en les présentant comme des prostituées. Elles dansent « toutes nues » et il n'y a rien « de plus lascif » que leurs postures et les gestes:

La nuit amène un spectacle analogue au goût dominant des peuples du midi, la danse des bayadères. Elle se fait au son du tâl et d'un petit tambour de terre. Lorsque les spectateurs sont généreux et peu scrupuleux, les domestiques se retirent et les danseuses paraissent toutes nues. Ce qu'on peut imaginer de plus lascif dans les postures et dans les gestes, accompagne alors leurs danses (Anquetil 363).

Pour revenir à notre anecdote sur la femme empoisonneuse, si l'on conçoit, à la suite de Todorov, que ce que nous appelons idéologie est « l'ensemble des attitudes et des idées partagées par la collectivité à un moment donné de son histoire » (Todorov 7), nous pouvons dire qu'une idéologie de la femme se met alors en place et c'est précisément à cause de cette vision stéréotypée de la femme que certains de nos voyageurs trouvent pertinent de prescrire le " suttisme » comme remède pour les femmes inconstantes. Notons aussi que cette explication ne trouve aucune mention dans les livres sacrés hindous. ${ }^{7}$ Qui plus est, le système judiciaire moghol, qui gardait un inventaire détaillé de tous les crimes et châtiments, ne parle pas de veuves hindoues ainsi punies parce qu'elles étaient des empoisonneuses (Banerjee « Disorderly wives » 137-173). L'absence de preuves dans les archives démontre

6 Voir à ce propos l'article d'Évelyne Berriot-Savadore «Le discours de la médecine et de la science. » Histoire des femmes en Occident XVIe XVIII ${ }^{e}$ siècles. Tome 3. Natalie Zemon Davis et Arlette Farge (éd.). Paris : Plon, $1991: 359-390$.

7 Voir à ce titre le livre de Simmi Jain Encyclopedia of Indian Women through the ages : Ancient times, Delhi, Gyan Publishing House, 2003 où l'auteur nous explique que les auteurs de Dharma Shastras et ceux de Manu et de Yajna Valkya ont précisé les devoirs d'une femme mariée ou d'une veuve dans la société. Pourtant aucun d'eux ne signale que ce serait louable pour une femme de se brûler au bûcher de son mari. Qui plus est, la pratique était fermement condamnée par les auteurs indiens de l'Antiquité. Banabhatta, auteur indien du VIIe siècle écrit: "The custom is a foolish mistake of stupendous magnitude committed under the reckless impulse of despair and infatuation... It does not ensure reunion since the wife who has uselessly sacrificied her life goes to hell reserved for suicides ». (Arvind Sharma Sati: Historical and phenomenological essays, Delhi, Motilal Banarsidas, 1988: 15). 
que cette anecdote est plutôt d'origine occidentale. Ce fait est confirmé par Frédéric Tinguely qui ajoute que certains voyageurs français comme Robert Challe étaient de l'opinion qu'il fallait imposer ce genre du châtiment aux femmes en occident afin de protéger les hommes :

Cette explication douteuse est notamment avancée à l'âge classique par le voyageur Charles Dellon ... Quant à Robert Challe, il ne croit pas à une telle cause mais n'en imagine pas moins les effets dissuasifs que pourrait produire l'importance du sati : « et si on obligeait en Europe les femmes à se brûler après la mort de leurs maris, les morts subites ne seraient pas si fréquentes ; et notre France n'aurait pas produit de mon temps des monstres tel qu'une Constantin, une Gorgibus, une Voisin, une Philbert, \& une infinité d'autres dont la Chambre ardente nous a rendu justice (Bernier 498)

Cet aveu laisse penser que l'anecdote de la femme empoisonneuse doit plutôt être vue comme un discours qui contribue à propager une image stéréotypée de la femme en général.

Notons que la superposition des modèles occidental et oriental de la femme imparfaite se retrouve également dans l'œuvre d'auteurs français bien connus aux $\mathrm{XVII}^{\mathrm{e}}$ et XVIII ${ }^{\mathrm{e}}$ siècles. Prenons comme exemple les Fables de Jean de la Fontaine. Le personnage féminin est pratiquement absent du deuxième recueil de cet ouvrage. Il y a bien sûr les dédicaces comme celle à Madame de Montespan ou même « le discours à Madame de la Sablière " où les dames sont mises sur un piédestal et qualifiées « d'Iris » ou " d'Olympe », mais l'image générale de la femme est celle d'un objet de ridicule. Dans la fable «Le Malmarié » (Livre VII, 2) par exemple, La Fontaine affirme que la bonté et la beauté sont deux vertus qui ne vont jamais de pair chez une femme :

Que le bon soit toujours camarade du beau

Dès demain je chercherai femme.

Mais comme le divorce entre eux n'est pas nouveau

Ne trouvez pas mauvais que je ne cherche point (La Fontaine 175).

L'apologue «Les femmes et le secret » (Livre VIII, 6) démontre que le beau sexe est synonyme de commérage tandis que dans "Les souhaits », le tisserand Mantharka qui avait gagné la faveur d'un esprit est dissuadé de tenir conseil avec sa femme car « là où une femme commande, la maison se ruine » (La Fontaine, 186). Le fabuliste ne condamne pas les actes de violence contre la femme. Dans «Le brahmane et le pot de farine » (livre V, 9), le mari songe à donner un coup depied à sa femme tandis que dans « Le brahmane et sa femme » (livre III, 17), l'homme coupe le nez de la femme qui lui est infidèle. La voix misogyne des personnages ou du narrateur n'est peut-être pas celle de l'auteur, mais les anecdotes comme celles de la femme empoisonneuse et les extraits littéraires laissent croire que les descriptions de la femme dans les divers genres littéraires ainsi que dans les récits de voyage, aux $\mathrm{XVII}^{\mathrm{e}}$ et $\mathrm{XVIII}^{\mathrm{e}}$ siècles étaient issus d'un même courant de pensée.

\section{Le suttisme : discours sur la femme vertueuse}


Nous avons vu que l'image de la femme durant l'Ancien Régime est ambivalente. D'une part les médecins et les moralistes sont de l'opinion qu'il fallait la maintenir en tutelle à cause de sa faiblesse physiologique et en raison de son infériorité morale. D'autre part, l'humanisme et le développement du culte marial redonnent à la femme une certaine liberté et surtout sa dignité de mère. C'est dans cette vision contrastée de la femme que s'insèrent les anecdotes sur le « suttisme » présentant la femme hindoue comme une sainte se soumettant à ce sacrifice avec la plus inflexible détermination. Dans tous ces exemples, la dévotion démesurée de la femme est glorifiée par nos voyageurs. Ils assurent que pour une femme hindoue, il n'y a d'autre divinité sur terre que son mari et, quand cette divinité terrestre meurt, elle doit donc le suivre dans la mort. Citons comme exemple l'anecdote de JeanBaptiste Tavernier qui montre bien la détermination des femmes hindoues :

Je me souviens d'une étrange action qui se passa un jour à Patna ville de Bengala. J'étais avec les Hollandais chez le gouverneur de la ville ... lorsqu'il entra dans la salle où nous étions une jeune femme parfaitement belle et qui ne pouvait guère avoir vingt-deux ans. Cette femme d'un ton ferme et résolu vint demander au Gouverneur la permission de se brûler avec le corps de son mari mort. Le Gouverneur touché de la jeunesse et de la beauté de cette femme tâcha de la détourner de sa résolution ; mais voyant que tout ce qu'il lui disait était inutile ... il s'avisa de lui demander si elle savait bien quel tourment c'était le feu, et s'il ne lui était jamais arrivé de se brûler à la main. Non, non lui répondit alors cette femme avec plus de fermeté qu'auparavant ... Quelques jeunes seigneurs qui étaient auprès de lui (le Gouverneur), le prièrent de vouloir bien qu'on éprouva cette femme et d'ordonner qu'on apporta un flambeau ... Dès que cette femme eût aperçu ce flambeau qui était bien allumé, elle courut au \devant, et tenant sa main ferme sur la flamme sans la moindre grimace et avançant même le bras jusqu'au coude qui fut tout incontinent grillé ... (Tavernier 391-392)

Tavernier informe ses lecteurs des lois qui interdisent aux femmes de se sacrifier sans obtenir la permission des gouverneurs de la ville où elles habitent. Cette loi est mise en place pour protéger les veuves contre cette coutume que le gouvernement trouvait exécrable. La jeune femme dans l'anecdote ci-dessus demeure ferme dans sa résolution de se brûler avec son mari. Elle fait preuve de sa détermination en brûlant sa main sur un flambeau sans montrer les moindres signes de douleur. Le comte de Modave, qui visite l'Inde un siècle plus tard, fait allusion à des sentiments semblables :

Je me rendis au lieu marqué sur les dix heures du matin et je vis qu'on charioit le bois destiné à faire le bûcher. Il n'y avait sur place que trois ou quatre brahmes ... la femme arriva .... plusieurs personnes l'aidèrent à descendre du chevalet on la porta dans la niche où elle s'assit à côté du corps de son mari. On en ferma alors l'entrée par des bois qu'on mit en travers avec des paquets de paille. Ensuite on amena l'enfant auquel on donna un brandon allumé; il fit le tour du bûcher et, après, il en alluma 
un côté ... Aussitôt que le bûcher fut en feu tous ces gentils se mirent à tourner autour en jetant des cris. Les instruments ajoutaient encore à ce vacarme ... L'image de cette femme est tellement gravée dans ma mémoire qu'elle ne s'en effacera jamais. Elle ne montra ni trouble ni agitation et s'acquitta de toutes ces affreuses cérémonies comme de la chose du monde la plus indifférente (Modave 174)

L'utilisation des phrases comme « elle ne montra ni trouble ni agitation » ou « elle s'acquitta de toutes ces affreuses cérémonies comme de la chose du monde la plus indifférente » démontre le stoïcisme de la femme hindoue et la consternation des voyageurs. L'abnégation de la sati devant l'ultime sacrifice et son dévouement sans bornes demeurent exemplaires.

En dernier lieu, nous voulons aussi souligner le fait que « la sati » ne prend jamais la parole (Spivak 2009). Ce sont les observateurs masculins qui parlent de cette pratique, rendant ainsi le sujet féminin muet. Le silence, d'après Pompa Banerjee, est vu comme synonyme de la chasteté féminine en occident. Ainsi, la femme indienne qui ne prend jamais la parole devient une figure exemplaire.

\section{Conclusion}

$\mathrm{Au}$ terme de cette étude, il est donc clair que tous les voyageurs n'utilisent pas l'anecdote de la sati pour promouvoir la représentation d'une altérité religieuse barbare. François Bernier et le comte de Modave arrivent à relativiser ce rite qui, par ailleurs, désigne métonymiquement une composante essentielle de l'hindouisme que les écrits religieux semblent fermement condamner comme étant le meurtre de soi. ${ }^{8}$

Le «suttisme» met aussi en scène un discours sur l'altérité féminine fracturée : vertueuses ou licencieuses, respectueuses des traditions ou affranchies, ces femmes hindoues sont souvent les victimes des idéologies des voyageurs. C'est donc au voyageur de décider du rôle de la femme.

\section{Bibliographie}

« Anecdote. » Trésor de la langue française. C.N.R.S, Institut de la langue Française. Paul Imbs (dir.). Paris, C.N.R.S, 1971.

Anquetil-Duperron et Abraham Hyacinthe. Voyage en Inde, 1754-1762. Paris : École Française d'Extrême Orient, 1997.

Banerjee, Pompa. « Disorderly wives, Poison, and the iconography of female murderers. » Burning Women: Widows, Witches and Early Modern European Travelers in India. New-York: Palgrave MacMillan, 2003: 137173.

---. Burning Women: Widows, Witches and Early Modern European Travelers in India. New-York: Palgrave, 2003.

Bernier, François. Un libertin dans l'Inde Moghole : Les Voyages de François

${ }^{8}$ Dans son article «Comment, the continuing invention of the sati tradition» Veena Talwar Oldenburg cite plusieurs auteurs indiens de l'Antiquité qui condamnent cette coutume. Ainsi Medhatithi écrivant au VII ${ }^{e}$ siècle appelle ce rite « adharma » ou aller à l'encontre de la religion. On trouve le même écho dans les travaux d'Aparaka et ceux de Devanabhatta. 
Bernier (1656-1669). Paris : Chandeigne, 2008.

Berriot- Salvadore, Evelyne. Les Femmes dans la société de la Renaissance, Genève : Librairie Droz, 1990.

Chakravorty-Spivak. Gayatri. « Subalternes peuvent-elles parler? » Trad. Jérôme Vidal. Paris : Éditions Amsterdam, 2009.

Cureau de la Chambre, Marin. L'Art de connoistre les hommes. Amsterdam : chez Jacques le Jeune, 1660.

Daniélou, Alain. La civilisation des différences. Paris-Pondichéry : Éditions Kailash, 2003.

---. Histoire de l'Inde. Paris : Fayard, 1983.

---. La civilisation des différences. Paris : Éditions Kailash, 2003.

Dryden, John. Aureng-Zebe, A Tragedy. London : Archibald Constable and Company, 1842.

Hartog, François. Le miroir d'Hérodote : Essai sur la représentation de l'Autre. Paris : Gallimard, 1981.

Hawley, John Stratton. Sati, The Blessing and the Curse. New-York: Oxford University Press, 2001.

Holwell, John. «The religious tenets of the Gentoos. The British Discovery of Hinduism in the Eighteenth Century. P.J. Marshall, P.J. (éd.). Cambridge: Cambridge University Press, 1970: 45- 106.

La Fontaine, Jean de. Fables. Paris : Livre de Poche, 1972.

Modave, Comte de. Voyage en Inde du comte de Modave 1773-1776. Jean Deloche (éd.) Paris : École Française d'Extrême-Orient, 1971.

Oldenburg, Veena Talwar. « Comment, the continuing invention of the sati tradition. » Sati, The Blessing and The curse: The Burning Wives in India, John Hawley (éd.). Stratton/ Oxford : Oxford University Press, 1994: 159172.

Platts, John T. A Dictionary of Urdu, Classical Hindi and English. New-Delhi: Munshiram Manoharlal, 1977.

Pyrard de Laval, François. Voyage de Pyrard de Laval aux Indes orientales (1601-1611). Geneviève Bouchon (éd.). Paris : Chandeigne, 1998.

Rabelais, François. Pantagruel. Paris : Pléiade, 1955.

Kieffer, Jean-Luc, Anquetil-Duperron : L'Inde en France au XVIII siècle. Paris : Belles Lettres, 1983.

Saïd, Edward. L'Orientalisme : l'orient créé par l'occident. Paris ; Seuil, 1980.

Shwab, Raymond. La Renaissance orientale. Paris : Payot, 1950.

Tavernier, Jean-Baptiste. Les six voyages en Turquie, en Perse et aux Indes Orientales. Paris : Gervais Clouzier et Claude Barbin, 1676.

Thévenot, Jean. Les voyages aux Indes orientales : contenant une description exacte de l'Indostan, des nouveaux mogols, et d'autres peuples et païs des Indes orientales, avec leurs mours et maximes, religions, fêtes, temples, pagodes, cimetières, commerce, et autre choses remarquables. Françoise de Valence (éd.)Paris : Champion, 2008.

Thevet, André. La Cosmographie du Levant. Frank Lestringant (éd.). Genève : Droz, 1985

Upreti, Nadini. The Myth of Sati: Some Dimensions of Widow Burning. Bombay: 
Himalaya Publishing House, 1991

Vijayan, Devika. Les récits de voyage français aux Indes orientales (XVII et XVIII ${ }^{e}$ siècles). Thèse de doctorat. Université de Waterloo : Canada, 2013. Weinberger-Thomas, Catherine. Cendres d'immortalité. La crémation des veuves en Inde. Paris : Seuil, 1996. 